\title{
Retrospective Evaluation of Efficiency and Safety of an Anterior Percutaneous Approach for Cervical Discectomy
}

\author{
Michael Schubert ${ }^{1}$, Susanne Merk ${ }^{2}$ \\ ${ }^{1}$ Apex-Spine Center, München, Germany \\ ${ }^{2}$ Vertebral Words, Gilching, Germany
}

\begin{abstract}
Study Design: Retrospective case series.
Purpose: The purpose of the study was to evaluate the efficiency and complication rate of a percutaneous anterior approach to herniated cervical disks with or without concomitant foraminal stenosis and/or spondylosis.

Overview of Literature: Recent publications reflect that minimally invasive procedures gain in importance in patients and spine surgeons as they are generally associated with less tissue damage and shorter recovery times. However, for anterior percutaneous cervical discectomy, very little data is available for relevant patient populations.

Methods: Charts from patients with herniated cervical disc confirmed by magnetic resonance imaging, mainly radicular symptoms and irresponsive to conservative treatment who underwent anterior percutaneous discectomy were evaluated retrospectively. All patients were asked to return questionnaires that included visual analogue scores (VAS), MacNab score as well as subjective satisfaction data 2 years after surgery.

Results: Ninety-five patients were included. There were no neurological or vascular complications; only one patient suffered from transient hoarseness. During the two years after surgery, 9 patients underwent reoperation. $90.5 \%$ of the patients returned the questionnaire at 2 years' follow-up. $87.7 \%$ of them reported excellent or good outcome, $11.1 \%$ rated results as fair and $1.2 \%$ as unsatisfactory. On average, arm and neck pain improved significantly by 6.1 points and 5.8 points respectively on a ten point VAS. $94.5 \%$ stated that they would choose the same procedure again.

Conclusions: This procedure has proved a safe and sufficient option for symptomatic cervical disk herniations with or without concomitant spondylosis and/or foraminal stenosis.
\end{abstract}

Keywords: Intervertebral disc displacement; Foraminotomy; Diskectomy, percutaneous; Neck pain

\section{Introduction}

Cervical discogenic pain is relatively frequent and a common cause of acquired disability in persons over 50 years of age [1]. About $80 \%$ of these cases respond to conserva- tive therapy. Only after all conservative options fail, surgery should be considered. Anterior cervical discectomy with fusion (ACDF) is still regarded as the gold standard surgical procedure for the treatment of cervical radiculopathy from a herniated disc by a majority of surgeons.

Received Apr 19, 2013; Revised Jul 26, 2013; Accepted Jul 28, 2013

Corresponding author: Susanne Merk

Vertebral Words, Kosthofstr. 35b, 82205 Gilching, Germany

Tel: +49-177-37-18-190, Fax: +49-911-30844-22047, E-mail: s.merk@vertebral-words.de 
As it may be associated with donor site morbidity or adjacent segment disease, alternative techniques like anterior cervical discectomy without fusion (ACD) and arthroplasty have been developed and gained popularity. These three techniques have been recommended as equivalent techniques providing rapid symptom relief by the current guidelines from the North American Spine Society [2].

Minimally invasive approaches have gained importance as they are in general associated with less tissue damage and shorter recovery times than traditional open techniques $[3,4]$. A number of different nucleoplasty techniques which only partly remove or shrink disc tissue with the aid of lasers, enzymes or coblation techniques have shown [5-12] conflicting results. The main reason for failure may bethe insufficient removal of pathological structures that compress the nerve roots; however, improper patient selection has also been discussed [13].

To the best of our knowledge there are no published data on relevant patient populations for percutaneous anterior cervical discectomy that can also be used in cases of sequestered disc herniations with or without foraminal stenosis or spondylosis.

We want to describe the surgical technique and present a retrospective analysis of a series of cases from our clinic.

\section{Materials and Methods}

\section{Ethics Committee}

The investigation was approved by the responsible review board of the State Medical Chamber of Bavaria (EthikKommission der Bayerischen Landesärztekammer). The approval number was 2011-108.

\section{Surgical technique}

Before surgery, individual anatomy as well as the exact position of herniated disc material and bony spurs leading to foraminal narrowing was evaluated in depth using magnetic resonance imaging (MRI)/computed tomography (CT) images. Surgery was then thoroughly planned to position instruments in a way that they reach all compressive material but do not damage any of the important visceral, vascular and neural structures in the neck.

The procedure was carried out with the patient in the supine position; the head was slightly extended and po- sitioned on a rubber ring (Fig. 1). The patient was kept under monitored anesthesia care (MAC) with a combination of midazolam and remifentanil for sedation and analgesia as well as continuous oxygen supply of at least $4 \mathrm{~L} / \mathrm{min}$ via nasal cannula. Midazolam and remifentanil were given intravenously and were adapted intraoperatively to individual patients' response. Remifentanil was also adjusted according to pain expected from the invasiveness of single procedural steps. Generally, the patient was kept at sedation level three in which the patient's eyes are closed but he/she can be roused on command. All standard monitoring equipment used in general anesthesia was applied, and an anesthesiologist was available in the operating room during the whole procedure.

The patient was aseptically prepared and draped on a radiolucent operating table. Anterior-posterior as well as lateral X-ray images were taken to identify the correct surgery level. The level was marked on the skin, and skin and subcutaneous tissues were locally anesthetized 3 centimeters from the midline. Access was always done from the ipsilateral side. With the index and middle fingers pulsating of the carotid artery was palpated, then the trachea and esophagus were displaced medially and the carotid artery laterally. The fingers were pushed to the front of the vertebral bodies to ensure a space for a spinal needle to approach the affected spinal disc (Fig. 2). Correct positioning of the needle was determined fluoroscopically and a discography with $1 \mathrm{~mL}$ of radiopaque dye was carried out. This was done to confirm that the patient's pain was generated by the disc and to verify the presence of annular tears.

After discography, a guide wire was pushed through the

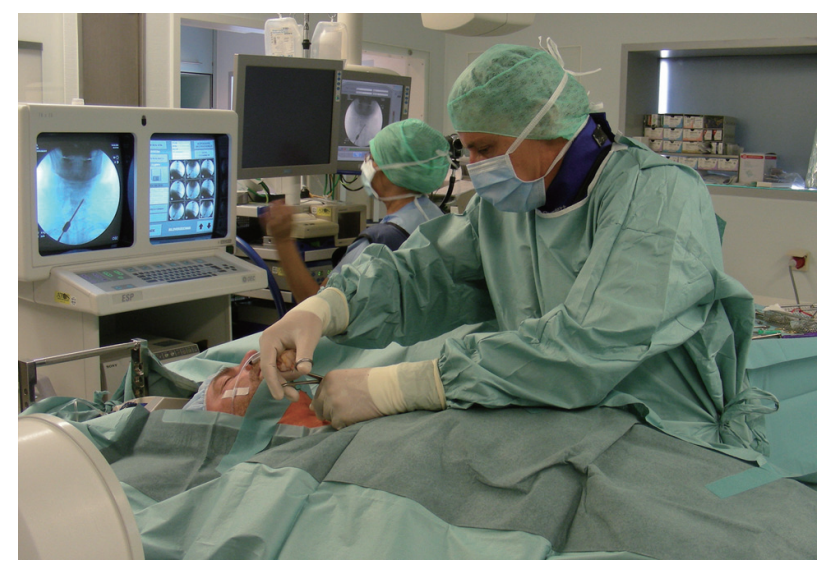

Fig. 1. View of the situation in the operating room during anterior percutaneous discectomy. 
needle, and the needle was removed. Sequential dilatation was done, and finally a three millimeter working cannula was pushed forward to the site of the prolapse (Fig. 3).

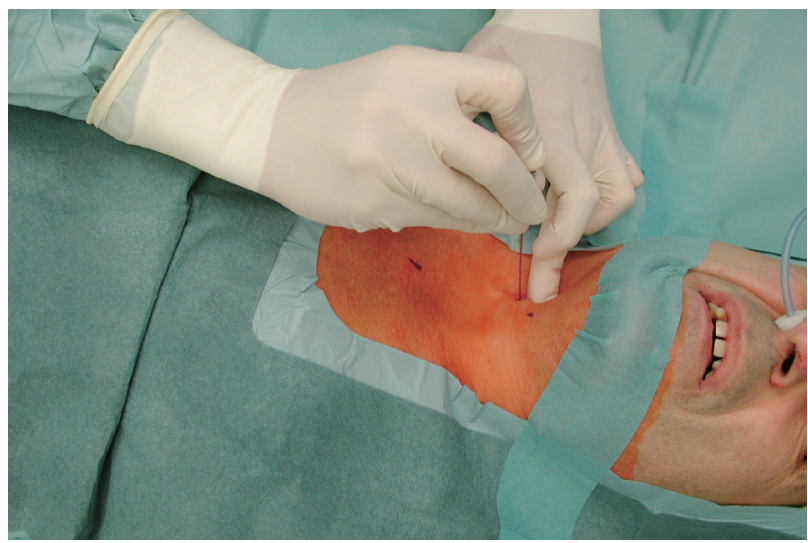

Fig. 2. From anterior and ipsilateral to the site of herniation, a spinal needle was introduced through a small skin incision to the affected disk level.
Through the working channel, foraminal decompression was additionally performed in all cases With a two millimeter reamer osteophytes were reamed, then with special minute forceps protruding and sequestered disc material was removed (Fig. 4). Correct positioning of the forceps was controlled fluoroscopically. The forceps had to be exactly at the place of expected disc herniation as analyzed from the MRI images.

The instruments were removed only when no more of the stained disc material was visible in fluoroscopy and no more material could be grasped-a careful tactile verification was done by rotating the forceps in different angles if the "surroundings" were really cleared. To conclude, the wound was closed with a stitch and covered with a strip.

The time needed for the surgery was about 30 minutes on average.

The patients were watched for two hours in the recov-
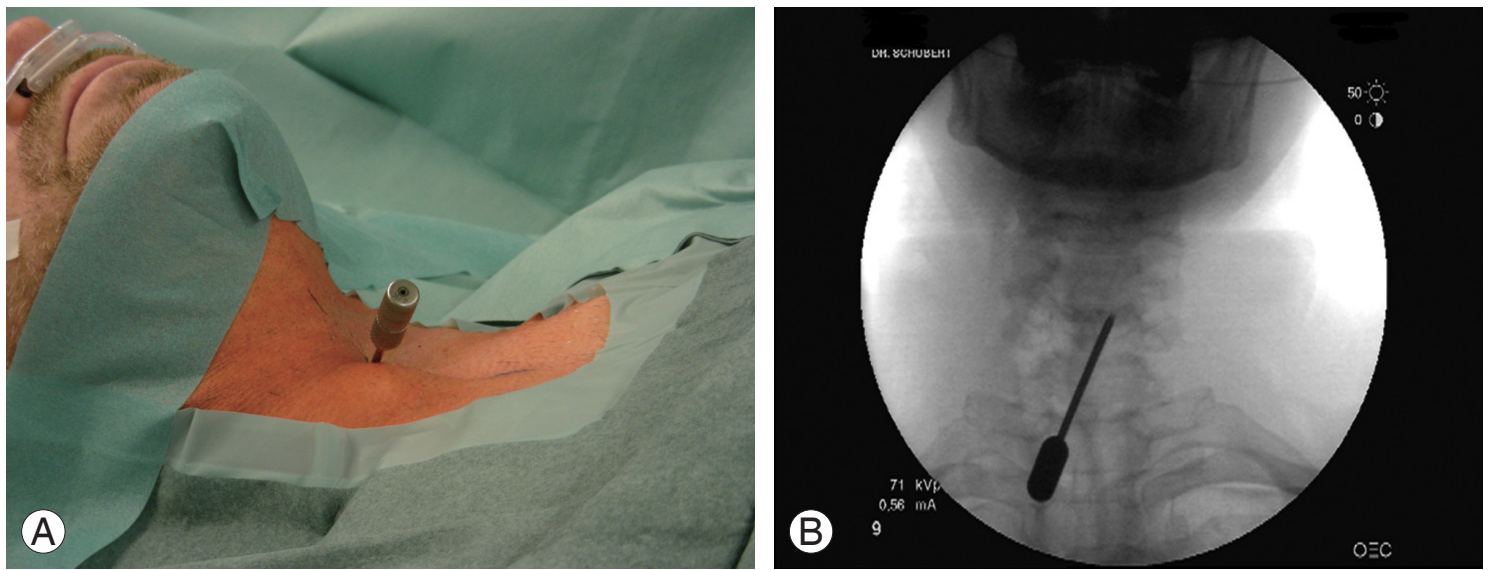

Fig. 3. (A) Sequential dilatation was done, and finally a three millimeter working cannula was pushed forward to the site of the prolapse. (B) Correct position was controlled fluoroscopically.
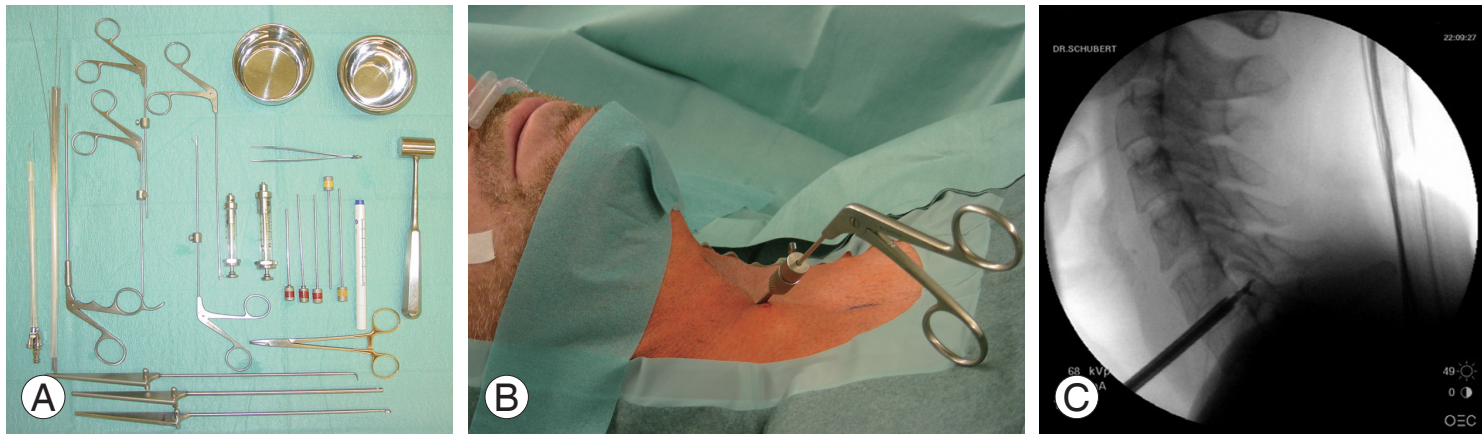

Fig. 4. With minute instruments, osteophytes as well as protruding and sequestered disk material was removed. (A) Set of instruments for percutaneous anterior discectomy. (B) Disk material was grasped with special forceps. (C) Its position was controlled fluoroscopically. 
ery room and then released home or to their hospital room without wearing a collar.

Most patients were treated on an outpatient base.

\section{Patients}

Data from patients that had undergone the percutaneous cervical discectomy described above during the period of September 2004 to January 2007 were evaluated. Patients that were eligible for this procedure had suffered from a radiologically (MRI or CT) confirmed contained or sequestered cervical herniated disc that correlated with clinical signs. The signs were severe cervicobrachialgia and/or neurological signs like relevant numbness or weakness of the upper extremities which had proven irresponsive to comprehensive conservative treatment over a period of at least 3 months. The patient's shoulder and arm pain had to be worse than neck pain to be considered for the intervention. Surgery was performed in patients with or without the presence of foraminal stenosis or spondylosis. Bilateral radiculopathy and central stenosis, however, were regarded as exclusion criteria. Also excluded from the present analysis were data from patients older than 60 years, those with previous cervical spine surgery or cervical myelopathy.

\section{Outcome criteria}

Charts of all patients were reviewed, and data were extracted regarding age and sex of patients, levels of treated herniations, perioperative complications as well as recurrences. Two years after surgery, a set of questionnaires was sent to all patients. The questionnaires comprised 10 point visual analogue scores (VAS) scores for arm and neck pain, MacNab criteria, the presence of numbness or weakness in the arms or hands and subjective satisfaction scores. For the presence of numbness or weakness, a fourgrade scoring matrix was chosen: patients could choose between "no numbness (weakness)", "less numbness (more strength)", "no change in numbness (strength)" and "worsened numbness (weakness)" when compared to before surgery. For subjective satisfaction regarding surgery outcome, one of the following four possibilities could be ticked: "very good", "good", "unchanged" or "worse". Patients could also indicate whether they would choose to have the same surgery again or if they had undergone any further intervention for their cervical disc
Table 1. Levels operated on within the 95 patients included in the analysis

\begin{tabular}{lcccc} 
Affected level & C3/4 & C4/5 & C5/6 & C6/7 \\
No. of patients & 4 & 15 & 44 & 44 \\
\hline 12 patients had two-level surgery. & & &
\end{tabular}

problem elsewhere during the two-year follow-up. If patients did not send questionnaires back within two weeks, we tried to reach them by phone. When their address was obsolete, we tried to find out their new address by phone, email or online directories.

\section{Data analysis}

Evaluation was done by directly summarizing the numerical parameters and calculating means with standard deviations where possible.

\section{Results}

\section{Patients and affected levels}

Between September 2004 and January 2007, 95 patients who had undergone percutaneous cervical discectomy were included in the present analysis. The mean age was 45 years and the most common affected levels were C5/6 and $\mathrm{C6} / 7$ ( $\mathrm{n}=44 \mathrm{each}$ ) (Table 1). Fifteen patients had a disc herniation at $\mathrm{C} 4 / 5$ and 4 at $\mathrm{C} 3 / 4$. Twelve patients were operated on two levels. Forty-nine men and 46 women underwent surgery.

\section{Complications and recurrences}

There were no infections, no bleedings and no neurological disturbances. One patient suffered from transient hoarseness. There had been three early recurrences within the first three months after surgery. Two of these patients were re-operated with the same percutaneous method and included in the two year follow-up. One rated his outcome as very good, the other as good. Within the whole follow-up period, 9 recurrences occurred. Three underwent percutaneous surgery again at our clinic, 5 underwent fusion-one of them at our institution-and one did not indicate what treatment he received. Of those patients that suffered from a relapse, seven returned the questionnaire after two years. Two of them rated their 
outcome as very good, two as good and three as unchanged.

\section{Clinical outcome}

Altogether 86 patients (90.5\%) returned the questionnaire after the two-year follow-up. As we are an internationally working institution, 7 of the 9 missing patients were from abroad and did not respond to our letters, emails or phone calls. The other two had changed their address and could not be further followed-up.

At baseline, 67 patients complained about paresthesias and 68 suffered from loss of strength.

Within the $90.5 \%$ of followed-up patients, on average, arm pain was significantly decreased by $6.1( \pm 2.1)$ points from $8.7( \pm 1.3)$ at baseline to $2.6( \pm 1.1)$ points at two year follow-up on the VAS $(p<0.001)$. Neck pain was reduced significantly by $5.8( \pm 2.1)$ points from 8.3 $( \pm 1.4)$ points before surgery to $2.5( \pm 1.6)$ points at 2 years $(p<0.001)$. In the vast majority of patients, paresthesias had vanished or improved: $43.3 \%(n=29)$ of patients had no and $47.8(\mathrm{n}=32)$ had less paresthesias compared to before surgery. $7.5 \%(n=5)$ found them unchanged and $1.5 \%(n=1)$ judged them worse than at baseline. The findings were similar for loss of strength $(n=68)$ : again most patients showed significant improvement. $32.4 \%(\mathrm{n}=22)$ completely returned to their previous levels of strength, $42.7 \%(n=29)$ regained some strength compared to before surgery, $23.5 \%(n=16)$ did not feel any increase of strength after surgery and one patient (1.5\%) felt that he was weaker after surgery than at baseline.

According to the MacNab criteria, outcome could be judged as excellent or good in $81.4 \%(\mathrm{n}=70)$ of the patients, only in $2.3 \%(\mathrm{n}=2)$ of the patients outcome was judged as poor ( $\mathrm{n}=86)$ (Fig. 5). The patients' subjective judgment on outcome was very good or good in $87.2 \%$ $(\mathrm{n}=75), 11.6 \%(\mathrm{n}=10)$ rated their condition as unchanged and one patient (1.2\%) found that the symptoms worsened $(n=86) .94 .2 \%(n=81)$ of the patients stated that they would choose this surgery again.

\section{Discussion}

The majority of the pathology that may compromise a nerve root is situated anterior to the dura: disc herniations and bony spurs of the vertebral bodies. An anterior approach hence suggests itself, and the ACD, ACDF or with an artificial disc replacement (ADR) have been recommended as comparable options with similar outcomes in the North American Spine Society guidelines for the

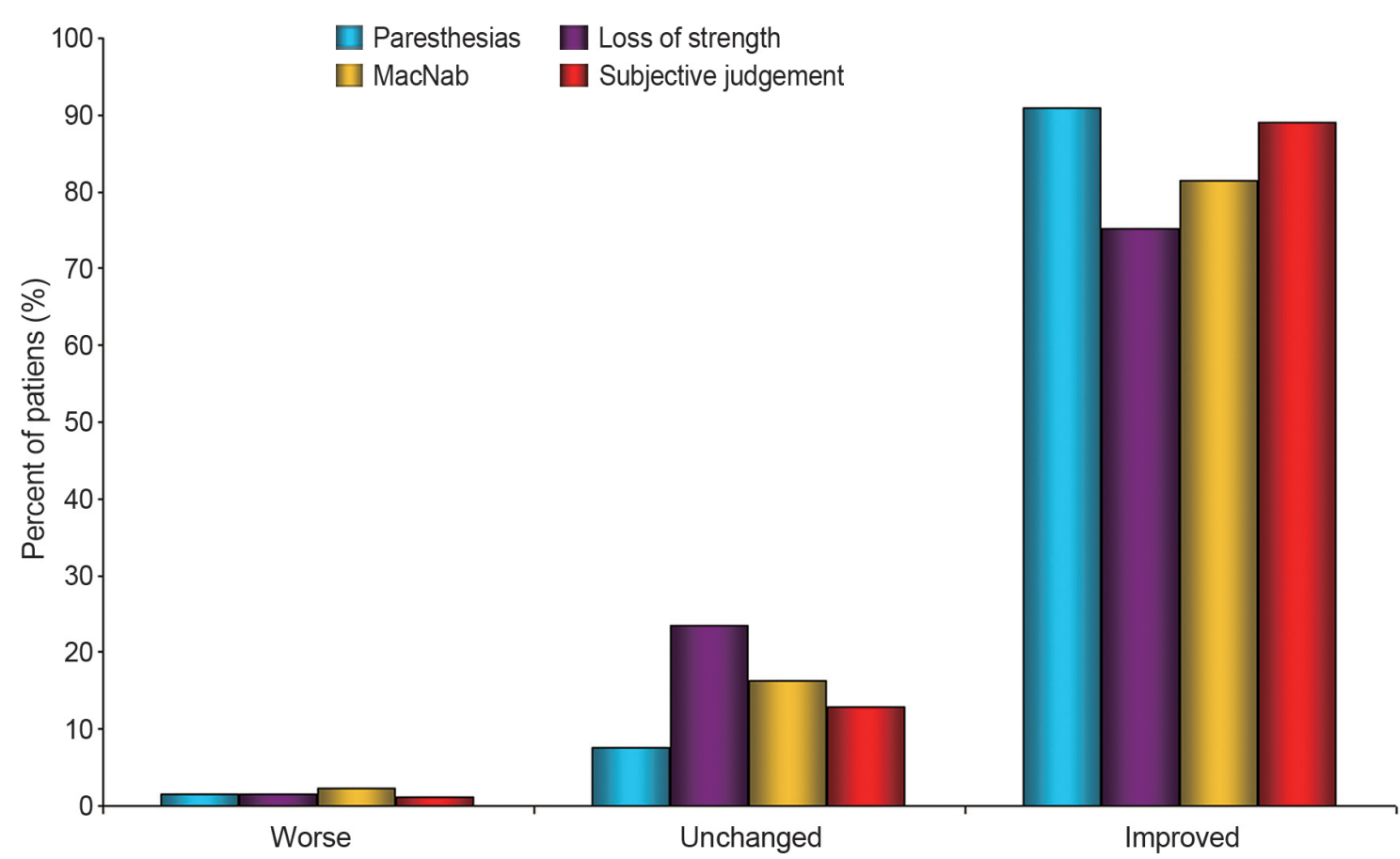

Fig. 5. Most patients showed favorable outcome at two-year follow-up. 87.7\% judged their condition as good or very good. 
treatment of single level cervical radiculopathy from degenerative disorders [2].

The presented anterior percutaneous approach to the spinal disc is similar to that used for discography. This technique was described in 1957 by Smith and Nichols [14] for the diagnosis of discogenic pain. It is widely used and generally considered safe, although there are some complications. They include infection, neural damage, hematoma, and damage to the carotid vessels, trachea, hypopharynx and esophagus. A review of 4,400 cervical discographies found a complication rate of only $0.16 \%$ of cases [15]. Although complications are rare, their potential severity requires expert techniques and experience for all percutaneous anterior cervical interventions.

A variety of complications associated with ACD and ACDF have been reported. Although they were mostly mild like transient dysphagia or dysphonia, severe complications like vertebral artery injuries have been described [16]. Very recently, cases of implant extrusion in ACDF and also in arthroplasty have been published $[17,18]$. The development of adjacent segment disease as a consequence of ACDF has been discussed controversially [19-21] but still remains a concern.

For the presented percutaneous technique-as opposed to conventional techniques-only a small amount of disc tissue has to be removed thus presumably preserving the functional unit of this segment. The observed complica- tion rate with the presented percutaneous technique was very low.

Adjacent segment disease is probably less of a concern as in ACDF as the segment, which is operated on, is believed to maintain its functionality. Although no long term MRIs have been taken systematically in our patient group, we did not observe any disc space collapse or severe narrowing of the disc space on available MRI/CT images (Fig. 6). Results published for a similar technique showed a mean loss of disc height of about $11 \%$ which was not associated with any significant effect on the therapeutic success. Sagittal alignment and segmental motion were well preserved [22].

Clinical outcomes were very good in our patient population-87.7\% were very satisfied or satisfied with the results of the intervention after two years, and $94.5 \%$ of the patients stated that they would choose this surgery again. Neurological signs were greatly reduced after surgery-patients suffered from significantly less arm pain, pareses and paresthesias. This is in line with previously published reports that found the surgery effective for the treatment of cervical radiculopathy in terms of pain reduction, disability, weakness, and numbness [23-26]. Also, significantly lower neck pain was reported after the intervention. Surgery for neck pain alone is generally not considered an adequate option. However, when neck pain is associated with radiculo- or myelopathy, surgical
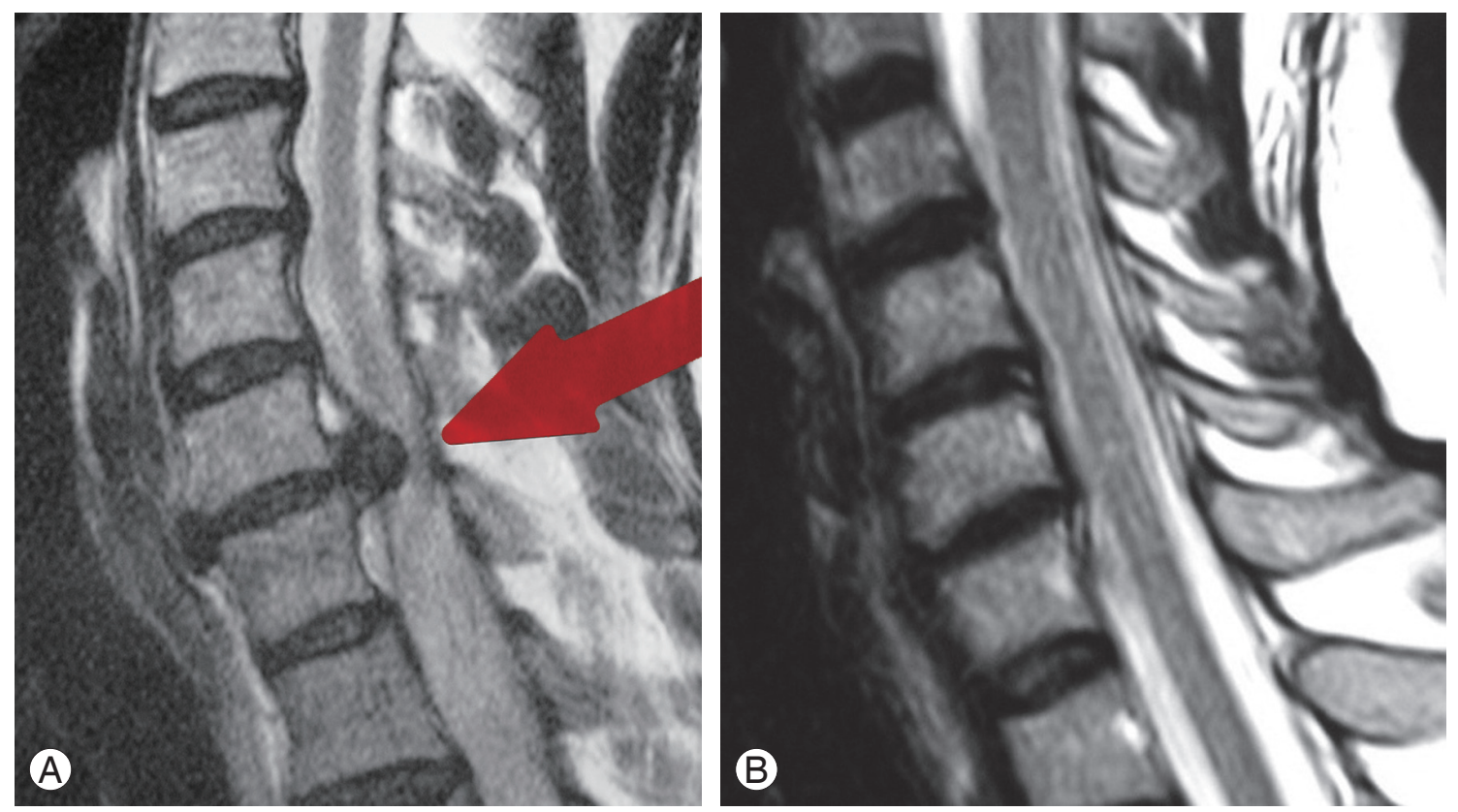

Fig. 6. Example of short-term radiologic outcome: (A) before and (B) 3 months after surgery. 
intervention has been reported to alleviate the symptoms $[25,26]$. Engquist et al. [26] recently published a randomized controlled trial comparing surgery plus physiotherapy to physiotherapy alone. The results suggested that the combined approach is superior to physiotherapy. Whereas there was only a clear trend in favor of surgery in terms of disability and arm pain reduction, statistical significance was reached for neck pain reduction. The mean intensity of neck pain reported in this study was also significantly reduced after surgery up to the final follow-up at 2 years.

Two of the three early recurrences had revision surgery with the same technique and approach from the same side as the first intervention. No verification was carried out if these two cases were true recurrent herniations or if patients suffered due to material that had not been fully removed during the primary operation. Early recurrences after lumbar discectomy have been recently analyzed by Cheng et al. [27]. They found that revisions within the early period after the first surgery are more common after minimally invasive techniques than after open procedures-about $46 \%$ of all reoperations were done within the first 6 months. This may be attributed to two possible causes: 1) hidden fragments are difficult to remove when they are beyond the working channel and 2) limited discectomy-although it produces overall better outcomehas been reported to be associated with more recurrences than aggressive removal of the disc [28]. Eventually, these results might translate to the situation within the cervical spine.

Due to different criteria for patient selection and differences in surgeons' experiences, it is not an easy task to compare the values of single surgical options. However, an evaluation of existing literature data on the recommended standard procedures (ACD/ACDF/ADR) and some of the more common minimally invasive techniques yielded a wide range of clinical outcomes. The success rates for percutaneous cervical discectomy ranged from $51.0 \%$ to $91.4 \%[6,7,9,13,22,29]$ and for ACD from $66 \%$ to $98 \%[5,30]$.

Whereas in all of the cited percutaneous techniques only patients with soft disc herniations and without bony spurs were included, the presented methods allows for the treatment of sequestered material and foraminal stenosis.

In our opinion, three main aspects may be considered as contributing factors leading to good clinical outcomes observed with the procedure: only a $3 \mathrm{~mm}$ working chan- nel is needed to gain access to pathology, the herniated contained or sequestered material is directly removed, and with small reamers, a foraminoplasty is done and hence osteophytes contributing to radiculopathy can be removed.

In an environment with growing needs to economize, clinical benefits have to be weighed against associated primary and secondary health care costs. The presented percutaneous technique has some cost saving potential: the procedure itself is shorter, postoperative time spent in the hospital can be assumedly greatly reduced in most cases, the recovery process is supposed to be much quicker and the patient might hence return earlier to work. As the intervention is done under local anesthesia, morbidity and costs stemming from adverse events associated with general anesthesia can be prevented.

Most important disadvantage to the technique is the long learning curve. The intervention is technically very demanding and should be carried out only by surgeons that have yearlong experience in standard spine procedures as well as percutaneous techniques to avoid serious complications.Also, the specific anesthesia protocol may not be routinely carried out in all centers. Close communication between surgeon and anesthesiologist is indispensable to adapt anesthetic medication according to the single steps of the operation.

Our study has some major limitations: it is a retrospective case series at a single center. Our first aim was hence to present the technique and to show that it can be carried out safely by an experienced surgeon and that it can lead to favorable clinical results in a relevant patient population. Another limitation is that no systematic radiological follow-up was carried out to better clarify degeneration at the index level due to the intervention.

Percutaneous cervical discectomy is now being done for several years at our institution and we regard it as a valuable and tissue-sparing alternative to ACDF or ADR in a carefully selected patient population.

To further judge how the technique compares to the more established procedures, studies with an appropriate randomized and controlled designed will certainly be indispensable.

\section{Conclusions}

The presented percutaneous cervical discectomy procedure has proved a safe and sufficient option for the treat- 
ment of cervical radiculopathy when performed by an experienced surgeon and in carefully selected patients. Further studies are needed to judge the value of the technique when compared to established procedures.

\section{Conflict of Interest}

No potential conflict of interest relevant to this article was reported.

\section{References}

1. Fehlings MG, Arvin B. Surgical management of cervical degenerative disease: the evidence related to indications, impact, and outcome. J Neurosurg Spine 2009;11:97-100.

2. Bono CM, Ghiselli G, Gilbert TJ, et al. An evidencebased clinical guideline for the diagnosis and treatment of cervical radiculopathy from degenerative disorders. Spine J 2011;11:64-72.

3. Kim CW, Siemionow K, Anderson DG, Phillips FM. The current state of minimally invasive spine surgery. Instr Course Lect 2011;60:353-70.

4. Schubert M, Hoogland T. Endoscopic transforaminal nucleotomy with foraminoplasty for lumbar disk herniation. Oper Orthop Traumatol 2005;17:641-61.

5. Rao PJ, Christie JG, Ghahreman A, Cartwright CA, Ferch RD. Clinical and functional outcomes of anterior cervical discectomy without fusion. J Clin Neurosci 2008;15:1354-9.

6. Bonaldi G, Baruzzi F, Facchinetti A, Fachinetti P, Lunghi S. Plasma radio-frequency-based diskectomy for treatment of cervical herniated nucleus pulposus: feasibility, safety, and preliminary clinical results. AJNR Am J Neuroradiol 2006;27:2104-11.

7. Chiu JC, Clifford TJ, Greenspan M, Richley RC, Lohman G, Sison RB. Percutaneous microdecompressive endoscopic cervical discectomy with laser thermodiskoplasty. Mt Sinai J Med 2000;67:278-82.

8. Harada J, Dohi M, Fukuda K, Nakazaki H, Koyama T, Abe T. CT-guided percutaneous laser disk decompression (PLDD) for cervical disk hernia. Radiat Med 2001;19:263-6.

9. Knight MT, Goswami A, Patko JT. Cervical percutaneous laser disc decompression: preliminary results of an ongoing prospective outcome study. J Clin Laser Med Surg 2001;19:3-8.
10. Krause D, Drape JL, Jambon F, et al. Cervical nucleolysis: indications, technique, results. 190 patients. J Neuroradiol 1993;20:42-59.

11. Miccoli P, Berti P, Raffaelli M, Materazzi G, Conte M, Faldini A. Minimally invasive approach to the cervical spine: a proposal. J Laparoendosc Adv Surg Tech A 2001;11:89-92.

12. Siebert W. Percutaneous laser discectomy of cervical discs: preliminary clinical results. J Clin Laser Med Surg 1995;13:205-7.

13. Ahn Y, Lee SH, Lee SC, Shin SW, Chung SE. Factors predicting excellent outcome of percutaneous cervical discectomy: analysis of 111 consecutive cases. Neuroradiology 2004;46:378-84.

14. Smith GW, Nichols P Jr. The technic of cervical discography. Radiology 1957;68:718-20.

15. Zeidman SM, Thompson K, Ducker TB. Complications of cervical discography: analysis of 4400 diagnostic disc injections. Neurosurgery 1995;37:414-7.

16. Daniels AH, Riew KD, Yoo JU, et al. Adverse events associated with anterior cervical spine surgery. J Am Acad Orthop Surg 2008;16:729-38.

17. Viezens L, Schaefer C, Beyerlein J, Thietje R, HansenAlgenstaedt N. An incomplete paraplegia following the dislocation of an artificial cervical total disc replacement. J Neurosurg Spine 2013;18:255-9.

18. Brenke C, Kindling S, Scharf J, Schmieder K, Barth M. Short-term experience with a new absorbable composite cage (beta-tricalcium phosphate-polylactic acid) in patients after stand-alone anterior cervical discectomy and fusion. Spine (Phila Pa 1976) 2013; 38:E635-40.

19. Bartolomei JC, Theodore N, Sonntag VK. Adjacent level degeneration after anterior cervical fusion: a clinical review. Neurosurg Clin N Am 2005; 16:57587.

20. Maldonado CV, Paz RD, Martin CB. Adjacent-level degeneration after cervical disc arthroplasty versus fusion. Eur Spine J 2011;20 Suppl 3:403-7.

21. Cho SK, Riew KD. Adjacent segment disease following cervical spine surgery. J Am Acad Orthop Surg 2013;21:3-11.

22. Ahn Y, Lee SH, Shin SW. Percutaneous endoscopic cervical discectomy: clinical outcome and radiographic changes. Photomed Laser Surg 2005;23:362-8.

23. Wirth FP, Dowd GC, Sanders HF, Wirth C. Cervical discectomy. A prospective analysis of three operative 
techniques. Surg Neurol 2000;53:340-6.

24. Gebremariam L, Koes BW, Peul WC, Huisstede BM. Evaluation of treatment effectiveness for the herniated cervical disc: a systematic review. Spine (Phila Pa 1976) 2012;37:E109-18.

25. Lofgren H, Johansen F, Skogar O, Levander B. Reduced pain after surgery for cervical disc protrusion/ stenosis: a 2 year clinical follow-up. Disabil Rehabil 2003;25:1033-43.

26. Engquist M, Lofgren H, Oberg B, et al. Surgery versus nonsurgical treatment of cervical radiculopathy: a prospective, randomized study comparing surgery plus physiotherapy with physiotherapy alone with a 2-year follow-up. Spine (Phila Pa 1976) 2013;38:171522.
27. Cheng J, Wang H, Zheng W, et al. Reoperation after lumbar disc surgery in two hundred and seven patients. Int Orthop 2013;37:1511-7.

28. McGirt MJ, Ambrossi GL, Datoo G, et al. Recurrent disc herniation and long-term back pain after primary lumbar discectomy: review of outcomes reported for limited versus aggressive disc removal. Neurosurgery 2009;64:338-44.

29. Zhou YC, Zhou YQ, Wang CY. Percutaneous cervical discectomy for treating cervical disc herniation: a report of 12 cases. J Tongji Med Univ 1994;14:110-3.

30. Matz PG, Ryken TC, Groff MW, et al. Techniques for anterior cervical decompression for radiculopathy. J Neurosurg Spine 2009;11:183-97. 